\title{
The Central Regions of Galaxies Hosting LINERs as Viewed by Chandra
}

\author{
H. Flohic ${ }^{1}$, M. Eracleous ${ }^{1}$, G. Chartas ${ }^{1}$, J. Shields ${ }^{2}$ and E. Moran ${ }^{3}$ \\ ${ }^{1}$ Dept. of Astronomy \& Astrophysics, Penn. State University, University Park, PA 16802 \\ ${ }^{2}$ Dept. of Physics \& Astronomy, Ohio University, Athens, OH 45701 \\ ${ }^{3}$ Astronomy Dept., Van Vleck Observatory, Wesleyan University, Middletiwn, CT 06459
}

\begin{abstract}
We have used Chandra archival observations of 19 galaxies hosting LINERs to explore the morphology and source population of their inner kiloparsec. Our goal was, in general, to determine the power source behind their nuclear X-ray emission and, in particular, to investigate the presence of an AGN. We find an AGN in 12 of the 19 galaxies in the sample. We also find that diffuse, thermal emission is common with properties very similar to what is found in normal galaxies. In 10 out of the 19 galaxies, the diffuce emission dominates the nuclear X-ray power. The X-ray point-source populations were studied by producing cumulative luminosity functions and their properties are also similar to what is found in normal galaxies.
\end{abstract}

\section{Introduction}

Low Ionization Nuclear Emission-Line Regions (LINERs) are defined based on their oxygen emission-lines ratios (Heckman 1980). They are common in nearby galaxies and are found in $30 \%$ of all galaxies, especially early types (Ho et al. 1997). A few mechanisms have been proposed to explain their line ratios (shocked gas, dense gas photoionized by hot stars or by X-rays from a LLAGN), but it is still unclear which mechanism is most relevant in LINERs. The goal of our study is to determine the power source of LINERs based on the X-ray properties of their central region; in particular, we have looked for indications for the presence of an AGN and/or unusual X-ray point source content.

\section{Sample and Analysis}

We selected all the LINERs in the Chandra Archives that were nearby $(d<25 \mathrm{Mpc})$ and observed for more than $15 \mathrm{ks}$ to ensure high spatial resolution and $S / N$, obtaining a sample of 19 objects. The host galaxies in this sample span a wide range of morphology and the relative numbers of LINER types are similar to those found in optical surveys.

For each galaxy we produced an image of the central kiloparsec in both the soft $\left(0.5^{-}\right.$ $2 \mathrm{keV})$ and hard $(2-10 \mathrm{keV})$ bands. We extracted the spectrum of all the detected sources using the package ACIS Extract, checked for the presence of a central point source, and fitted the spectrum of the brightest sources to determine their nature. We also extracted the spectrum of the diffuse emission in the central kiloparsec of each galaxy. Since the diffuse emission of individual galaxies did not produce enough counts to perform a meaningful fit, we stacked the spectra of spiral and elliptical galaxies separately.

\section{Results}

The galaxies in the sample have, on average, three X-ray point sources in their inner kiloparsec with a maximum of 12 sources. In 12 of the 19 galaxies we find a central X-ray 

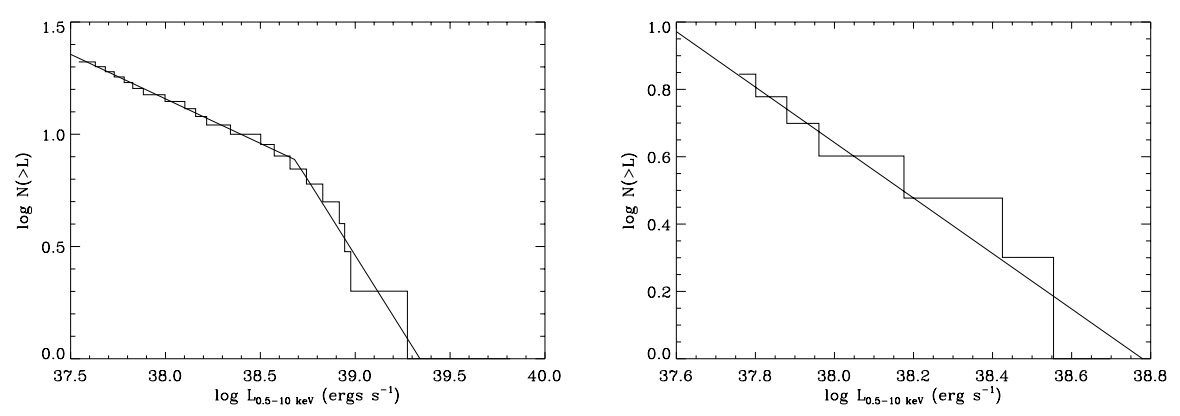

Figure 1. Cumulative luminosity function for the points sources in the elliptical galaxies (left, 21 sources) and for the spiral galaxies(right, 7 sources). The continuous lines show single or broken power-law fits to the data.

point source, which is often hard and always radio-loud. The typical X-ray luminosity of these AGN candidates is $L_{0.5-10 \mathrm{keV}}=10^{38}-10^{39} \mathrm{erg} \mathrm{s}^{-1}$. We compared the luminosity of the candidate AGNs to that of a $2 . .5$ diameter circle (similar to the area used in optical spectroscopic surveys) and found that the contribution of the AGN candidate to the X-ray luminosity of this region can vary between 30 and $100 \%$. We calculate that $L_{B o l} / L_{E d d}$ is between $10^{-7}$ and $10^{-4}$ for these AGNs (assuming a black hole mass of $\left.10^{7} M_{\odot}\right)$.

Diffuse emission is common in the central region of galaxies hosting LINERs (it is found in $70 \%$ of the sample). We created stacked spectra for the spiral and elliptical galaxies separately, which are well fitted by a thermal plasma model $(\mathrm{kT} \sim 0.6 \mathrm{keV})$ plus a power-law (photon index 1.3-1.5). This is very similar to the spectrum of diffuse emission found in normal galaxies (Sarazin et al. 2001, Sivakoff et al. 2004).

In Figure 1 we show separate cumulative luminosity function for all the non-nuclear sources found in the central region of the spiral and elliptical galaxies. These are well fitted by power-laws (broken for the elliptical galaxies) with indices very similar to those found for normal galaxies (Sarazin et al. 2001, Colbert et al. 2004).

\section{Conclusions}

LINERs are a heterogeneous population. $60 \%$ of the LINERs in our sample possibly harbor an AGN. In 70\% we find significant diffuse emission in the inner kiloparsec. Some LINERs have significant contributions from both the diffuse emission and the possible AGN to the X-ray luminosity of the innermost 2.5 . The X-ray point-source populations found in the central region of galaxies hosting LINERs do not differ from those found in normal galaxies. Thus, even though a significant fraction of the X-ray power is produced by stellar processes, a recent starburst does not seem to be involved.

Acknowledgements: This work was supported by NASA through grant AR4-5010A from the Smithsonian Astrophysical Observatory.

\section{References}

Colbert, E. et al. 2004, ApJ, 602, 231

Heckman, T. M 1980, A\&A, 87, 152

Ho, L. C., Filippenko, A. V. \& Sargent, W. L. W. 1997c, ApJ, 487, 568

Sarazin, C. L., Irwin, J. A. \& Bregman, J. N. 2001, ApJ, 556,533

Sivakoff, G. R., Sarazin, C. L. \& Irwin, J. A. 2003, ApJ, 599, 218 